\title{
Holographic characterization and tracking of colloidal dimers in the effective-sphere approximation
}

\author{
Lauren E. Altman, ${ }^{1}$ Rushna Quddus, ${ }^{2}$ Fook Chiong Cheong, ${ }^{3}$ and David G. Grier ${ }^{1}$ \\ ${ }^{1}$ Department of Physics and Center for Soft Matter Research, \\ New York University, New York, NY 10003, USA \\ ${ }^{2}$ Department of Chemistry, New York University, New York, NY 10003, USA \\ ${ }^{3}$ Spheryx, Inc., 330 E. 38th Street, \#48J, New York, NY 10016, USA
}

\begin{abstract}
An in-line hologram of a colloidal sphere can be analyzed with the Lorenz-Mie theory of light scattering to measure the sphere's three-dimensional position with nanometer-scale precision while also measuring its diameter and refractive index with part-per-thousand precision. Applying the same technique to aspherical or inhomogeneous particles yields the position, diameter and refractive index of an effective sphere that represents an average over the particle's geometry and composition. This effective-sphere interpretation has been applied successfully to porous, dimpled and coated spheres, as well as to fractal clusters of nanoparticles, all of whose inhomogeneities appear on length scales smaller than the wavelength of light. Here, we combine numerical and experimental studies to investigate effective-sphere characterization of symmetric dimers of micrometer-scale spheres, a class of aspherical objects that appear commonly in real-world dispersions. Our studies demonstrate that the effective-sphere interpretation usefully identifies dimers in holographic characterization studies of monodisperse colloidal spheres. The effective-sphere estimate for a dimer's axial position closely follows the ground truth for its center of mass. Trends in the effective-sphere diameter and refractive index, furthermore, can be used to measure a dimer's three-dimensional orientation. When applied to colloidal dimers transported in a Poiseuille flow, the estimated orientation distribution is consistent with expectations for Brownian particles undergoing Jeffery orbits.
\end{abstract}

\section{INTRODUCTION}

Holographic particle characterization [1] is a fast and robust technique for measuring the size and refractive index of colloidal spheres and has a wide variety of applications in basic research [2 5 , industrial materials analysis [6] and medical diagnostics [5, 7. As illustrated in Fig. 11(a), holographic particle characterization uses inline holographic video microscopy [1, 8] to record holograms of individual colloidal particles and then fits those holograms to predictions of the Lorenz-Mie theory of light scattering [9 11] to track the the particle in three dimensions and to measure its size and refractive index. Originally developed for analyzing homogeneous isotropic spheres, holographic particle characterization also has been applied to dimpled spheres [12, porous spheres [13, 14, coated spheres [15, and fractal aggregates of colloidal nanoparticles [3, 16, 17]. In all cases, the particles are analyzed with a generative model based on the theory of light scattering by homogeneous spheres. The properties obtained in this way therefore should be interpreted as parameters of an effective-sphere model. Effective-sphere properties then can be related to actual properties such as porosity [13, 14, or fractal dimension [3, 17] through effective-medium theory [13, 14, 18].

The success of the effective-sphere model hinges on the assumption that inhomogeneities in the particles' structure or composition are evenly distributed throughout the volume of the particle on length scales smaller than the wavelength of light. This is not the case, for example, when micrometer-scale colloidal spheres aggregate into dimers, trimers and similar small clusters. Holograms formed by such clusters can be analyzed quanti- tatively using suitable generative models for light scattering by clusters [19, 20. Such detailed analysis, however, is orders of magnitude slower than the corresponding effective-sphere measurement. Small clusters, moreover, figure prominently in holographic characterization measurements of real-world colloidal dispersions, such as the example in Fig. 1(b). The ability to identify and interpret holograms of such clusters within the effectivesphere framework therefore would create immediate applications in areas as diverse as semiconductor processing [16] and medical testing [7].

Here, we use numerical studies to assess the utility of the effective-sphere model for interpreting holograms of small colloidal clusters composed of micrometer-scale spheres. These studies demonstrate that small clusters can be clearly identified in dispersions of monodisperse colloidal spheres. The ability to identify and count colloidal dimers provides a basis for measuring their relative abundance, which is useful in such applications as detecting agglutination in bead-based medical assays [5, 7].

When effective-sphere analysis is applied to dimers, the observed distribution of single-particle properties is found to be parameterized by the dimers' orientation relative to the optical axis. Holographic characterization in the effective-sphere approximation therefore enables realtime tracking of colloidal dimers' orientations. We apply this technique to experimental studies of silica dimers transported in a Poiseuille flow down a microfluidic channel and demonstrate that the observed distribution of dimer orientations is consistent with expectations for Jeffery orbits. For symmetric colloidal dimers, therefore, the effective-sphere model appears to offer quantitative insights into the particles' trajectories and orientations. 

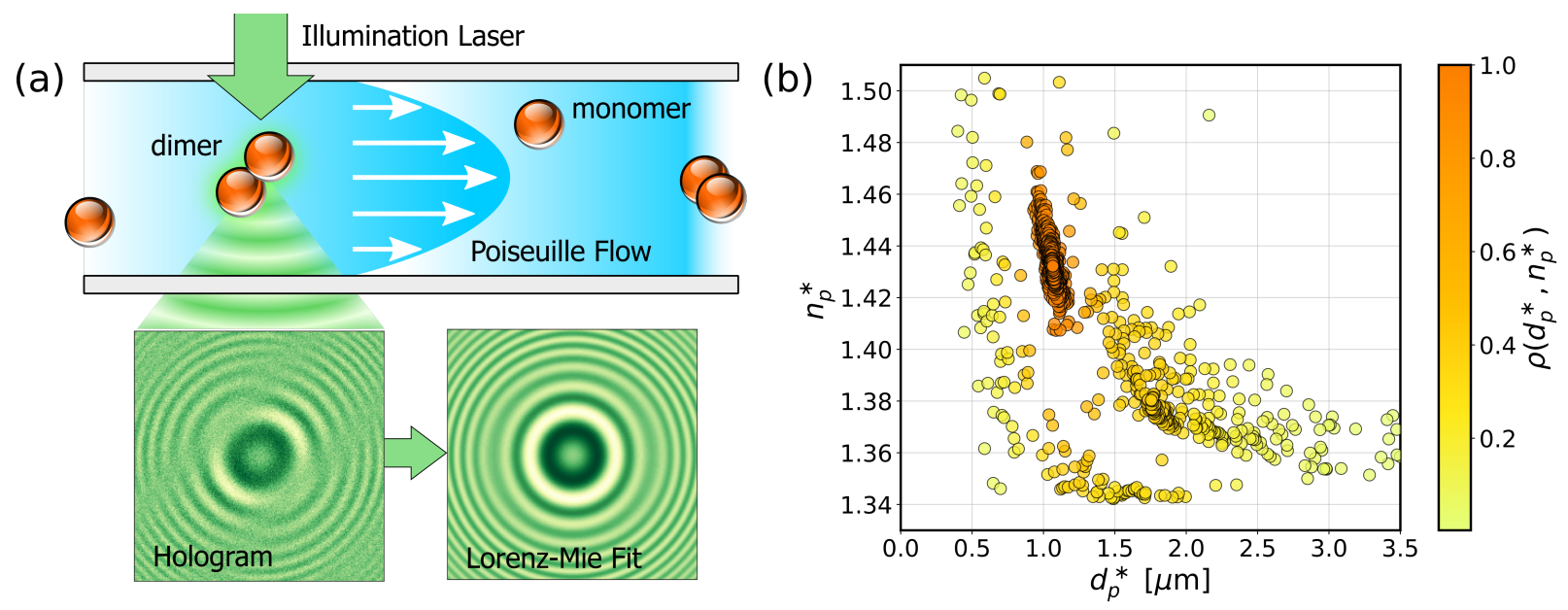

FIG. 1. (a) Schematic representation of holographic characterization of colloidal spheres and clusters in a microfluidic channel. Particles, including clusters, passing through the illuminating laser beam scatter light that interferes with the rest of the laser beam to create a hologram. Fitting a hologram to a generative model based on the Lorenz-Mie theory of light scattering yields measurements of the particle's effective diameter, $d_{p}^{*}$, and refractive index, $n_{p}^{*}$. (b) Scatter plot of single-particle properties measured for a nominally monodisperse sample of micrometer-diameter silica spheres that have been functionalized for a medical diagnostic test. Each plotted point represents the measured properties of one particle and is colored by the relative probability density of observations, $\rho\left(d_{p}^{*}, n_{p}^{*}\right)$. Clusters of points are likely to represent distinct types of particles, with at least three clusters being evident here.

\section{HOLOGRAPHIC PARTICLE CHARACTERIZATION}

Holographic particle characterization is an application of in-line holographic video microscopy [8, in which the sample is illuminated with a collimated laser beam. A colloidal particle entering the beam scatters some of the light, and the scattered light interferes with the rest of the beam in the focal plane of a conventional optical microscope. The microscope magnifies the interference pattern and relays it to a video camera that records its intensity. Each image in the resulting video stream is a hologram of the particles in the observation volume and encodes information about their three-dimensional positions, as well as their sizes, shapes and compositions. This information can be extracted by fitting recorded holograms to a generative model for the image-formation process. The standard implementation [1] models the incident beam as a unit-amplitude monochromatic plane wave at frequency $\omega$, whose electric field,

$$
\mathbf{E}_{0}(\mathbf{r}, t)=e^{i k z} e^{-i \omega t} \hat{x}
$$

is linearly polarized along $\hat{x}$ and propagates along $\hat{z}$. The wavenumber, $k=2 \pi n_{m} / \lambda$, depends on the laser's vacuum wavelength, $\lambda$, and the refractive index of the medium, $n_{m}$, and is related to the frequency through the standard dispersion relation, $\omega=c k$, where $c$ is the speed of light in the medium.

This plane wave illuminates a particle located at $\mathbf{r}_{p}$ relative to the center of the microscope's focal plane, which scatters some of the light. The time-averaged intensity pattern recorded by the camera may be modeled as a superposition of the incident and scattered waves,

$$
b(\mathbf{r})=\left|\hat{x}+e^{-i k z_{p}} \mathbf{f}_{s}\left(k\left(\mathbf{r}-\mathbf{r}_{p}\right)\right)\right|^{2},
$$

where $\mathbf{f}_{s}(k \mathbf{r})$ is the Lorenz-Mie scattering function for the particle 9 -11]. Experimentally recorded holograms are corrected for the dark count of the camera and normalized by the intensity distribution of the illumination to facilitate comparison with Eq. (2).

For the particular case of a spherical scatterer, the Lorenz-Mie scattering function is parametrized by the sphere's diameter, $d_{p}$, and refractive index, $n_{p}$. Analyzing a single-particle hologram with Eq. (2) involves optimizing these two parameters in addition to the particle's three-dimensional position, $\mathbf{r}_{p}$. The only calibration constants required for this analysis are the vacuum wavelength of the laser, $\lambda$, the magnification of the microscope and the refractive index of the medium, $n_{m}$, all of which can be measured independently. For the studies that follow, we choose $\lambda=445 \mathrm{~nm}$ and a system magnification of $120 \mathrm{~nm} /$ pixel for compatibility with commercial holographic particle characterization instruments (Spheryx, Inc., xSight). We furthermore assume that the medium has the refractive index of water at the imaging wavelength, $n_{m}=1.340$.

Standard software implementations of holographic particle characterization 21 25] can localize and characterize a sphere within 50 milliseconds on a desktop computer workstation. Validation experiments on colloidal size standards demonstrate that each such fit can resolve the diameter of a micrometer-scale sphere with a precision of $\pm 2 \mathrm{~nm}$ [26. Measurements on emulsion droplets demonstrate precision and reproducibility in the refrac- 
tive index of \pm 1 part per thousand [27. The former is fine enough to detect the formation of a molecular coating on a bead through the associated change in the bead's diameter [5, 28. The latter is useful for distinguishing different types of beads on the basis of their composition [29.

\section{THE EFFECTIVE-SPHERE MODEL}

\section{A. Inhomogeneous Spheres}

The standard Lorenz-Mie analysis treats the scatterer as an isotropic homogeneous sphere. A porous sphere, by contrast, is composed of a matrix of refractive index $n_{0}$ arranged at volume fraction $\Phi$ within the body of the particle. The remainder of the sphere's volume is filled with the medium at refractive index $n_{m}$. This inhomogeneous particle still may be treated as a homogeneous medium if its inhomogeneities are distributed uniformly through its volume on a length scale smaller than the wavelength of light. In that case, its effective refractive index, $n_{p}^{*}$, may be related to $n_{0}, n_{m}$ and $\Phi$ through Maxwell Garnett effective medium theory [13, 14, 18,

$$
n_{p}^{*}=n_{m} \sqrt{\frac{1+2 \Phi L\left(n_{0} / n_{m}\right)}{1-\Phi L\left(n_{0} / n_{m}\right)}},
$$

where the Lorentz-Lorenz factor is

$$
L(m)=\frac{m^{2}-1}{m^{2}+2} .
$$

The effective-sphere model described by Eq. (3) has been found to agree quantitatively with experimental results for mesoporous colloidal spheres [14, which should satisfy all of the assumptions underlying the model. More surprisingly, Eq. (3) also yields quantitative results for fractal clusters of dielectric nanoparticles [3, 17] even though those clusters are neither uniformly porous nor ideally spherical. These experimental [3] and simulation studies [17] suggest that the effective diameter, $d_{p}^{*}$, that emerges from Lorenz-Mie analysis roughly bounds the irregular cluster and that the effective refractive index, $n_{p}^{*}$, is consistent with the volume fraction of material, $\Phi$, within that bounding sphere.

\section{B. Symmetric Colloidal Dimers}

The hologram of a colloidal cluster could be analyzed by suitably generalizing the scattering function, $\mathbf{f}_{s}(k \mathbf{r})$, to account for the cluster's composition, geometry and orientation [19]. Introducing these additional adjustable parameters, however, reduces the likelihood that the fits will converge successfully, increases the measurement's sensitivity to imaging imperfections and very substantially increases the measurement time [30]. In many cases

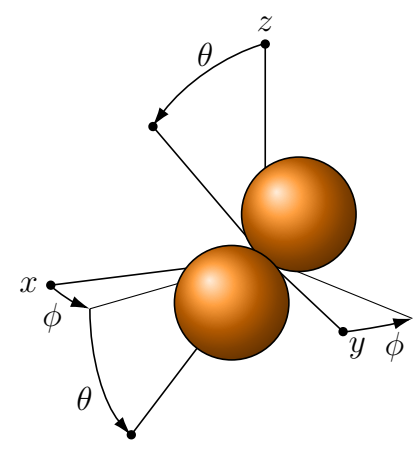

FIG. 2. Geometry of symmetric dimers used for computing cluster holograms. A pair of spheres initially is aligned with the $\hat{x}$ axis and then is rotated through two Euler angles, $\phi$ and $\theta$.

of interest, furthermore, the detailed geometry of a small colloidal cluster is of less interest than the ability to differentiate clusters from monomeric spheres. This is the case, for example, in medical diagnostic tests where agglutination of functionalized beads can indicate successful detection of the target analyte 31]. We therefore seek to understand how the standard effective-sphere analysis accommodates the geometry and orientation of small colloidal clusters.

We approach this problem through numerical experiments, generating synthetic holograms of colloidal clusters then applying effective-sphere analysis to obtain the clusters' effective positions, diameters and refractive indexes. These effective parameters then can be interpreted in light of the ground-truth properties of the clusters, thereby providing guidance for interpreting experimental particle-characterization data.

We compute the scattering functions for colloidal clusters with cluster $\mathrm{T}$ matrix theory [32, 33] using the HoloPy API [30, then apply Eq. 22 to compute ideal holograms, and finally add $5 \%$ Gaussian noise to simulate experimental recordings. We analyze those synthetic holograms with the pylorenzmie implementation of holographic particle characterization. Results obtained by analyzing synthetic holograms then can be compared directly with experimental observations on colloidal dispersions that are known to include aggregates.

Figure 2 shows the geometry used for computing synthetic holograms of colloidal dimers. A symmetric dimer centered at the origin can be parameterized by the monomers' diameter, $d_{p}$, and the Euler angles, $\phi$ and $\theta$. The polar angle $\theta=0$ corresponds to the dimer lying with its axis in the imaging plane. At $\theta=\pi / 2$, the dimer is aligned with the optical axis. The azimuthal angle $\phi=$ 0 aligns the dimer with the light's axis of polarization. With these definitions, the centers of the two monomers are situated at $\mathbf{r}_{ \pm}= \pm \frac{1}{2} d_{p} \hat{n}$ relative to the dimer's center, $\mathbf{r}_{p}$, where $\hat{n}=(\cos \phi \cos \theta, \sin \phi \cos \theta, \sin \theta)$ is the dimer's orientation unit vector. The dimer's description is completed with choices for the spheres' refractive index, $n_{p}$, and the axial position, $z_{p}$, of the dimer's center 

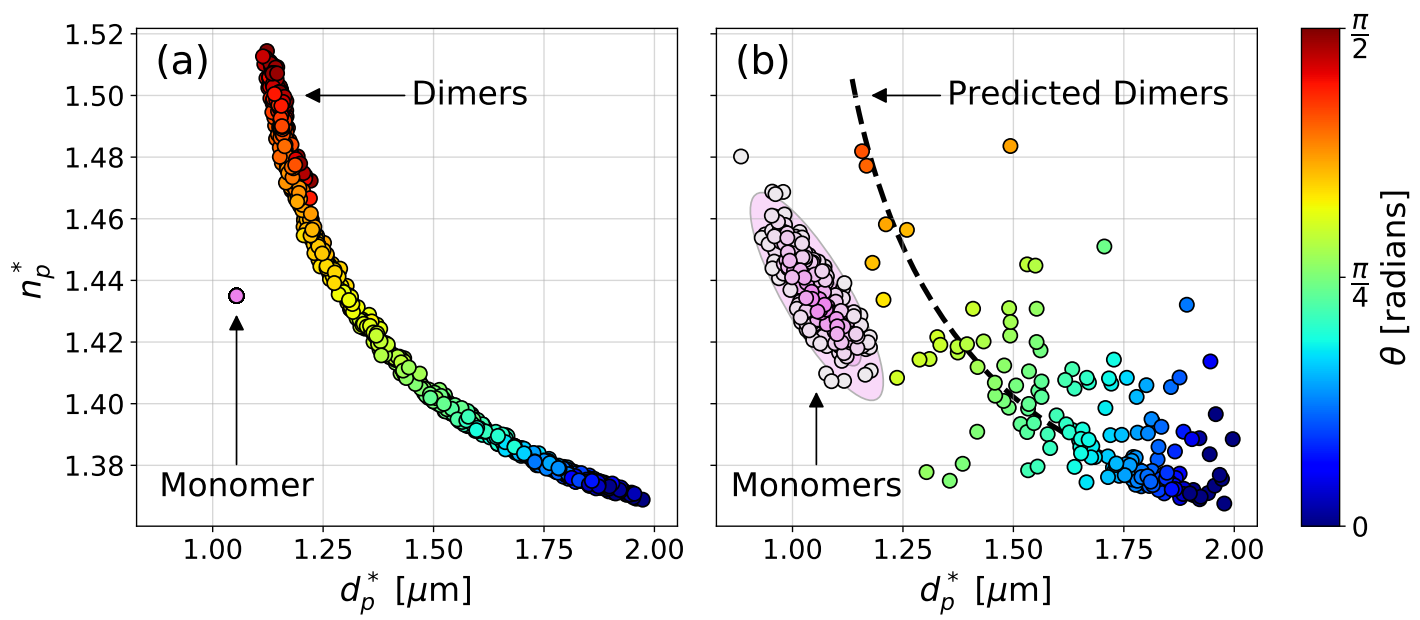

FIG. 3. Effective-sphere interpretation of a (a) simulated and (b) experimental holograms of colloidal monomers and dimers. Simulations are performed for symmetric dimers composed of silica spheres $\left(n_{0}=1.435\right)$ with diameter $d_{0}=1.054 \mu \mathrm{m}$ at varying polar angle $\theta$. Individual points are colored by $\theta$. The values for $d_{0}$ and $n_{0}$ are selected to mimic the experimental system. The dimers' effective diameters, $d_{p}^{*}(\theta)$, and refractive indexes, $n_{p}^{*}(\theta)$, are fitted to the phenomenological model from Eq. (4) that is plotted as the dashed black curve in (b). Holographic characterization data are segmented into monomers and dimers. Data points identified with dimers are colored by the angle $\theta$ of closest approach to the predicted dimer curve.

relative to the microscope's focal plane. A synthetic hologram defined by the set of parameters, $\left(d_{p}, n_{p}, z_{p}, \theta, \phi\right)$, is analyzed with Eq. (2) to obtain the effective-sphere descriptors, $d_{p}^{*}$ and $n_{p}^{*}$, as well as an estimate for the dimer's axial position, $z_{p}$.

\section{Effective Dimer Size and Refractive Index}

Results of a typical numerical study are presented in Fig. 3(a). Each point in this scatter plot represents the effective diameter and refractive index of a single particle, either a monomeric sphere or a randomly oriented dimer. The spheres' properties are selected to mimic the monodisperse silica probe beads used for holographic immunoassays [7]. Values obtained for the spheres' properties are found to be independent of axial position over the range $20 \mu \mathrm{m}<z_{p}<120 \mu \mathrm{m}$, with the lower bound being set by the spatial resolution of the imaging grid and the upper bound by the images' signal-to-noise ratio. The extended cluster of points in Fig. 3(a) represents results for colloidal dimers with random orientations in the range $0 \leq \theta \leq \frac{\pi}{2}$ and $0 \leq \phi \leq \pi$, and with axial positions in the range $50 \mu \mathrm{m} \leq z_{p} \leq 95 \mu \mathrm{m}$. Each point in the dimer distribution is colored by the ground truth value for $\theta$.

As might be expected, dimers lying in the imaging plane appear nearly twice as large as their component spheres. This is consistent with previous applications of effective-sphere analysis to irregular colloidal particles [3, 17] that found the estimated parameters to correspond to an average over a bounding sphere. The effective refractive index of the in-plane dimer therefore is closer to that of the medium because the volume fraction of the silica spheres within the effective sphere is just $\Phi=\frac{1}{4}$. Equation (3) then sets the expected lower bound for the observed refractive index to be $n_{p}^{*}>1.36$.

The aspherical structure of a dimer is far less apparent when the dimer is aligned with the optical axis, $\theta=\frac{\pi}{2}$. An aligned dimer scatters more light than a single sphere and thus appears to have a higher refractive index. Its effective diameter, however, is only slightly larger than that of a monomer. The effective-sphere model from Sec. III does not account for these observations presumably because the dimer's inhomogeneities appear on a scale larger than the wavelength of light, which means that the particle's orientation strongly influences its light-scattering properties. The apparent agreement for the in-plane dimer is all the more remarkable when viewed in this light.

It should be noted at this point that the identity of any individual colloidal particle as a dimer would not be evident on the basis of these single-particle characterization measurements. The distribution of particle characterization data in the $\left(d_{p}^{*}, n_{p}^{*}\right)$ plane, however, has a characteristic shape that can be recognized in the broader distribution of a mixed sample, such as the example in Fig. 1(b). If we identify the principal peak in those data with a population of monodisperse spheres, then the set of larger particles at lower refractive index trace out a curve that can be recognized as arising from dimers.

\section{Dependence on Dimer Orientation}

The results in Fig. 3(a) suggest that the distribution of effective properties recorded for dimers is simply parameterized by $\theta$ and does not depend significantly on 

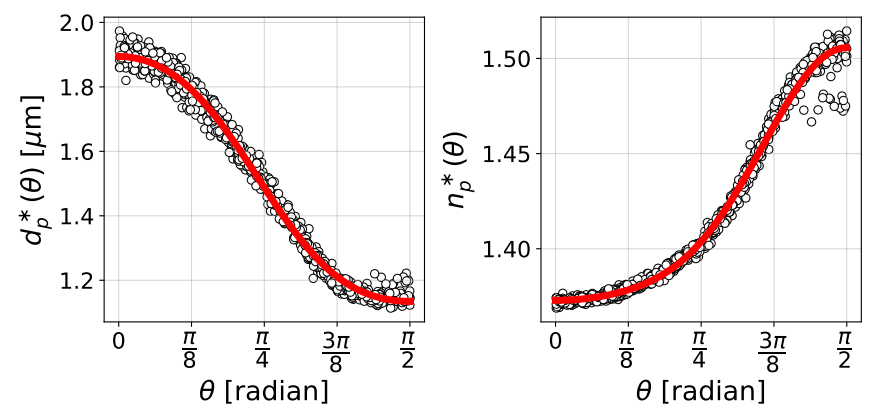

FIG. 4. Dependence of the effective-sphere diameter, $d_{p}^{*}(\theta)$, and refractive index, $n_{p}^{*}(\theta)$, on dimer orientation, $\theta$. Discrete points are the data for simulated dimers plotted in Fig. 3(a). Solid (red) curves are fits to Eq. (4).

$\phi$. Lacking a theoretical model for $d_{p}^{*}(\theta)$ and $n_{p}^{*}(\theta)$, we instead fit the measured effective-sphere results to polynomials in $\sin ^{2}(\theta)$,

$$
\begin{aligned}
& d_{p}^{*}(\theta)=\sum_{j=0}^{N} d_{j}^{*} \sin ^{2 j}(\theta) \\
& n_{p}^{*}(\theta)=\sum_{j=0}^{N} n_{j}^{*} \sin ^{2 j}(\theta),
\end{aligned}
$$

which respect the symmetries of the system and should yield the effective-sphere predictions for the in-plane properties, $d_{0}^{*}$ and $n_{0}^{*}$.

The results presented in Fig 4 are obtained with $N=3$ and yield $d_{0}^{*}=1.9 \mu \mathrm{m}$ and $n_{0}^{*}=1.37$. The fit value for the aligned dimers' diameter is slightly smaller than the diameter of the bounding sphere, and the fit value for the refractive index is correspondingly higher. The computed data in Fig. 3(a) are plotted over the parameterized curve obtained from these fits. The same fit also is reproduced as a dashed curve in Fig. 3(b), where it is compared with experimental data on comparable spheres.

\section{E. Effective-Sphere Measurement of Axial Position}

The same effective-sphere analysis used to obtain a particle's effective diameter and effective refractive index also yields its axial position, $z_{p}$, relative to the microscope's focal plane. For micrometer-scale colloidal spheres, the measured value of $z_{p}$ is found to have a precision of $\pm 5 \mathrm{~nm}$ over the entire accessible axial range 34 .

Figure 5 shows that effective-sphere analysis yields surprisingly good values for symmetric dimers' effective positions, with errors consistently smaller than $1 \mu \mathrm{m}$ over a range extending to nearly $100 \mu \mathrm{m}$. These measurements tend to underestimate the dimers' height above the imaging plane by an amount that increases with height and depends on the dimers' orientations. Errors tend to be smallest for dimers aligned with the imaging plane or aligned with the optical axis, and largest for $\theta=45^{\circ}$.
Outliers from these trends are likely to represent convergence of the nonlinear least-squares fit into a secondary minimum of the error surface [35].

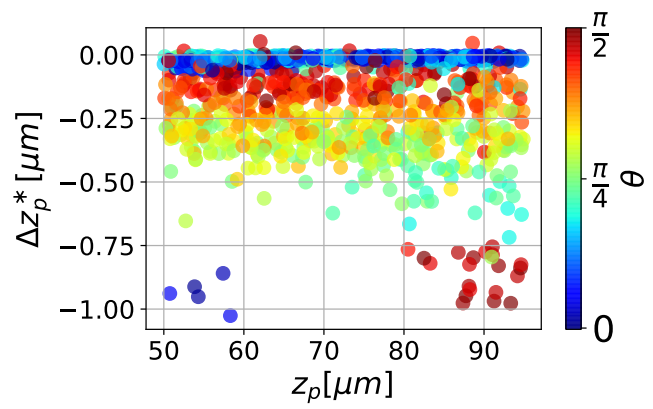

FIG. 5. Error in the effective-sphere axial position, $z_{p}^{*}$, for the simulated symmetric dimers from Fig. 3(a) as a function of true axial position, $z_{p}$. Each point is colored by the dimer's orientation, $\theta$. Effective-sphere analysis tends to underestimate $z_{p}$, with the errors tending to be greater for dimers aligned at $\theta=45^{\circ}$ from the imaging plane.

\section{APPLICATION TO EXPERIMENTAL DATA}

\section{A. Identifying Dimers}

When analyzing experimental holographic characterization data such as the results in Fig. 11(b), the identities of individual particles are not known a priori. Sharp peaks in the $\left(d_{p}^{*}, n_{p}^{*}\right)$ plane are likely to represent populations of monodisperse spheres. In this particular case, the sample is known to include a population of $1 \mu \mathrm{m}$-diameter silica spheres coated with antibodies for a medical diagnostic test. The band of low-index objects extending to the lower size limit of the instrument are likely to represent protein aggregates [6] and bacterial contaminants such as E. coli [36]. Based on the foregoing discussion of colloidal dimers, we propose that the remaining broad cluster of objects represents clusters of the monomers, including symmetric dimers.

We automatically and objectively distinguish the three populations of particles by segmenting the data into three categories using the Gaussian mixture model, as implemented in scikit-learn 37. Figure 3(b) reproduces data from the monomer and cluster categories, with violet points corresponding to monomers and rainbowcolored points corresponding to clusters. The third category, tentatively identified with protein aggregates and bacteria, has been omitted.

After segmentation, we find that the monomers have population-average properties $d_{p}=(1.054 \pm 0.009) \mu \mathrm{m}$ and $n_{p}=1.435 \pm 0.005$. These values were used for the simulations that are plotted in Fig. 3(a). The dashed curve in Fig. 3(b) shows the distribution of effectivesphere diameters, $d_{p}^{*}(\theta)$, and refractive indexes, $n_{p}^{*}(\theta)$, 
predicted for symmetric colloidal dimers.

As anticipated, the data points identified with colloidal clusters are distributed along the numerically predicted curve for symmetric dimers. This validates the assignment of those data points as dimers. This capability can be put to good use in counting the numbers of monomers and dimers in a sample, which in turn can be used to measure the rate of dimerization.

The predicted dimer curve is parameterized by polar angle, $\theta$. We can assign a value for $\theta$ to each measured data point by identifying the closest point along the predicted curve. The data points for identified colloidal clusters are colored accordingly in Fig. 3(b). The measured orientations are not uniformly distributed along the predicted curve, but rather are concentrated near $\theta=0$. This trend is expected for rod-like particles tumbling in a shear flow, and is addressed in Sec. IV C. We first assess the nature of the fluid flow in the microfluidic channel and the particles' spatial distribution within that flow.

\section{B. Axial Coordinates and Flow Profiling}

Holographic characterization measurements yield three-dimensional trajectories of colloidal particles that can be used to map flow profiles in the fluid medium 28]. The data in Fig. 6(a) show the measured flow speed, $v_{p}\left(z_{p}\right)$, as a function of the particles' axial positions, $z_{p}$, for the experimental data from Fig. 1 (b). The data points are colored by their classification as either monomers or dimers from Fig. 3(b). Being co-dispersed in the same fluid, both populations of particles trace out the same parabolic profile characteristic of Poiseuille flow in a rectangular channel. Fitting to a parabola yields estimates for the position of the upper and lower walls of the channel, which are plotted as horizontal dashed lines in Fig. 6(a). The $H=53 \mu \mathrm{m}$ separation between the walls is consistent with specified dimensions of the xCell microfluidic channel (Spheryx, Inc.) used for this measurement. The width of the measured distribution of flow speeds reflects variations in the actual flow speed encountered in xSight measurements.

The flow profile reported by dimers is consistent with the flow profile mapped by the monomers. This observation further validates the conclusion drawn from simulation that effective-sphere characterization of symmetric dimers yields accurate values for the dimers' axial positions. The fluid's true flow profile serves as an absolute reference for both populations of particles. The submicrometer systematic downward bias in the dimers' axial position anticipated from Fig. 5 is too small to resolve in the measured distributions of axial positions, $\rho\left(z_{p}\right)$, plotted in Fig. 6(a).

Monomers and dimers appear with roughly equal probability at all heights in the channel suggesting that they are present in this sample at roughly equal concentrations. The concentrations of both classes of particles decrease sharply within $10 \mu \mathrm{m}$ of either wall presumably be- cause of shear-induced migration mediated by collisions [39]. Having mapped the shear flow in the channel and the locations of particles within that flow, we can interpret the observed distribution of dimer orientations.

\section{Orientation Distribution and Jeffery Orbits}

Aspherical particles tend to rotate in shear flows, their orientation vectors tracing out trajectories called Jeffery orbits [40]. The combination of shear forces, inertia, viscous drag and diffusion cause aspherical particles, such as dimers, to undergo complex gyrations. Minimizing drag favors trajectories that align dimers with the axis of vorticity [41, which lies in the imaging plane for our system. We expect, therefore, to see more dimers aligned with $\theta=0$ than with $\theta=\pi / 2$, which is consistent with the measured orientation distribution, $P(\theta)$, plotted in Fig. 3(b). This probability density is obtained with a Gaussian kernel density estimator for the distribution of dimer orientation angles obtained by projecting the effective-sphere estimates for $d_{p}^{*}$ and $n_{p}^{*}$ onto the computed curves for symmetric dimers, $d_{p}^{*}(\theta)$ and $n_{p}^{*}(\theta)$.

Given the measured flow speed on the midplane of $v_{0}=3 \mathrm{~mm} / \mathrm{sec}$, the channel height of $H=53 \mu \mathrm{m}$ and the depletion of particles near the wall, we would expect dimers to experience a maximum effective shear rate of $\dot{\gamma} \leq 2 v_{0} / H \approx 100 \mathrm{~s}^{-1}$. The rotational diffusion coefficient for colloidal dimers is similar to that of a prolate spheroid of 2:1 aspect ratio. For dimers of micrometerdiameter spheres diffusing in water, we therefore expect [42, 43, $D_{r} \approx 0.4 \mathrm{~s}^{-1}$. These estimates suggest that our system has a maximum rotational Péclet number $\Gamma_{\max }=\dot{\gamma} / D_{r} \approx 200$.

The discrete points plotted in Fig. 6(b) are experimental results for $P(\theta)$ reported by Zöttl et al. 38 for micrometer-scale colloidal rods being transported in a flow geometry very similar to ours. The orientation angles in this case were estimated with conventional microscopy by measuring the projected lengths of the colloidal rods. In this case, $\dot{\gamma} \leq 16 \mathrm{~s}^{-1}$ and $D_{r}=0.21 \mathrm{~s}^{-1}$, yielding $\Gamma_{\max }=76$. One data point at $P(0)=2.5$ falls outside the plot range of Fig. 6(b). Despite the factor of three difference in rotational Péclet numbers between this study and ours, the reported values for $P(\theta)$ agree remarkably well.

While quantitative agreement between the two experimental data sets may be coincidental, common features in the shape of $P(\theta)$ highlight general aspects of particles' tumbling trajectories that are not yet fully explained 38 . In both data sets, for example, the distribution of particle orientations does not decrease monotonically from $\theta=0$, but rather has a deep minimum between $\theta=0$ and a second peak around $\theta=\pi / 8$. This minimum is notably absent from the hydrodynamic simulations reported by Zöttl et al. [38.

The Fokker-Planck equation describing the rotational advection and diffusion of a Brownian particle in a uni- 
(a)

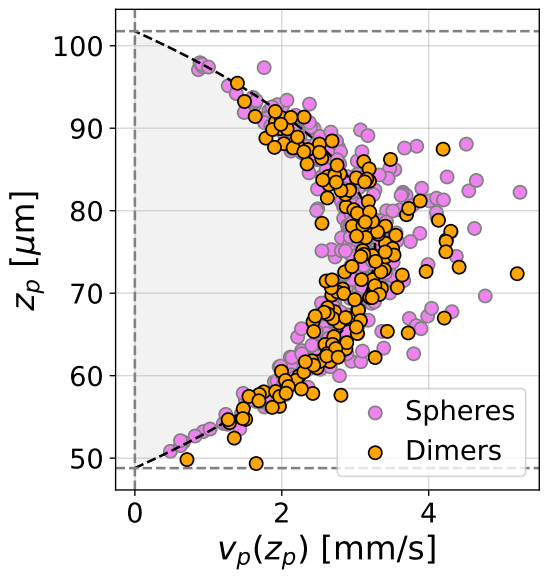

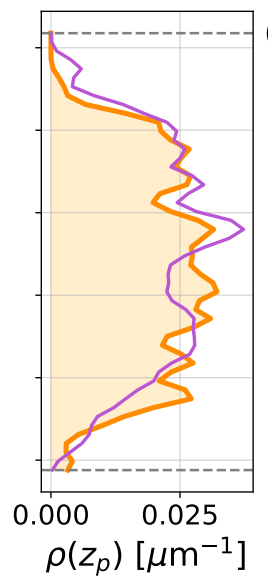

(b)

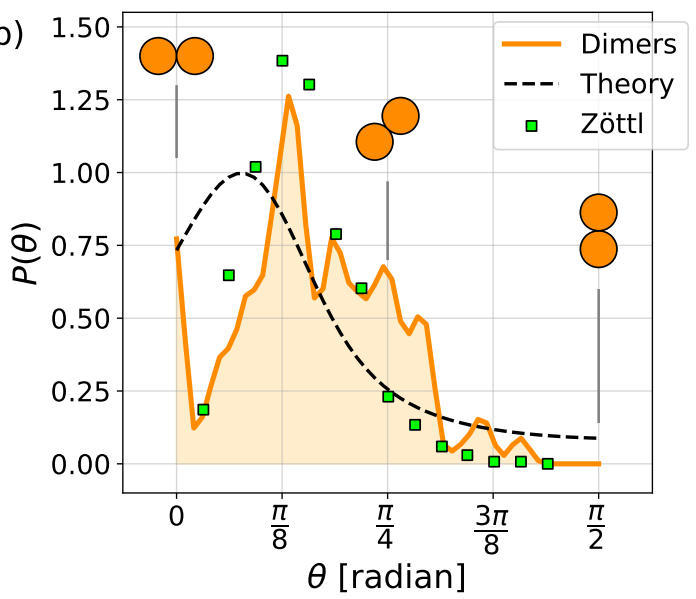

FIG. 6. (a) The holographically measured flow profile, $v_{p}\left(z_{p}\right)$, for particles identified as dimers agrees with the flow profile for individual spheres co-dispersed in the same system. This agreement validates the effective-sphere estimate of $z_{p}$ for the dimers. The dashed curve is a fit to the parabolic profile expected for Poiseuille flow, which also yields estimates for the axial positions of the upper and lower walls, indicated by dashed lines. The projected distribution of axial positions, $\rho\left(z_{p}\right)$, shows that spheres and dimers are both concentrated near the midplane of the channel. (b) Angular orientation distribution, $P(\theta)$, for dimers (orange) estimated by projecting measured effective-sphere characterization data onto the computed curves for $d_{p}^{*}(\theta)$ and $n_{p}^{*}(\theta)$. Discrete (green) points reproduce experimental results on Brownian rods in a Poiseuille flow from Zöttl et al. 38 . The dashed curve is the prediction of Eq. (6) for $\Gamma_{\max }=50$.

form shear flow has a steady-state solution [4],

$$
Q(\theta, \Gamma)=\frac{f(\pi, \Gamma) \int_{0}^{\theta} f\left(\theta^{\prime}, \Gamma\right) d \theta^{\prime}+\int_{\theta}^{\pi} f\left(\theta^{\prime}, \Gamma\right) d \theta^{\prime}}{z(\Gamma) f(\theta, \Gamma)}
$$

where $f(\theta, \Gamma)=\exp (\Gamma u(\theta))$ and $u(\theta)=\frac{\theta}{2}-\frac{\sin 2 \theta}{4}$. The normalization constant, $z(\Gamma)$, can be determined numerically and is approximately given by

$$
z(\Gamma) \approx \frac{\left(\frac{3 \pi}{2}\right)^{2}+\Gamma}{1+\Gamma} e^{\pi \Gamma}
$$

Equation (5) is appropriate for a rod-like particle in the uniform shear of a Couette flow. The Poiseuille flow mapped in Fig. 3(a) has a shear rate that varies linearly from roughly $\dot{\gamma}=200 \mathrm{~s}^{-1}$ near the walls to zero on the midplane. So long as $\dot{\gamma}$ does not vary appreciably over the dimensions of the particle, however, the orientation distribution function may be approximated as an average of the Couette result. If, furthermore, the dimers are distributed uniformly in the flow, the average over $z$ can be replaced by an average over $\Gamma$, yielding

$$
P(\theta)=\frac{1}{\Gamma_{\max }} \int_{0}^{\Gamma_{\max }} Q(\theta, \Gamma) d \Gamma
$$

The dashed curve in Fig. 6(b) is the prediction of Eq. (6) for $\Gamma_{\max }=50$, which is smaller than the rotational Péclet numbers in either our study or by Zöttl et al. 38. Like the experimental results, this distribution is peaked near $\theta=\pi / 8$. Unlike the experimental results, the theoretical distribution does not feature a peak at $\theta=0$, nor is the predicted peak in $P(\theta)$ as sharp as the experimentally observed peak. Lower values of $\Gamma_{\max }$ emphasize diffusion and yield flatter distributions than are seen experimentally. Larger values sharpen the peak, but move it to smaller angles.

It is possible that these discrepancies could be resolved by accounting for inertial effects [1] or steric interactions between the particles and the walls. Regardless of their origin, the experimental observation of peaks in $P(\theta)$ lend credence to the proposal that holographic particle characterization provides reasonable estimates for the axial orientation angle, $\theta$, of symmetric colloidal dimers, even when analyzed in the effective-sphere approximation. Effective-sphere analysis may be useful, therefore, in resolving persistent discrepancies between the theory of shear-induced tumbling and experimental observations.

Holograms, such as the example in Fig. 1(a) also encode information on a dimer's azimuthal orientation angle, $\phi$. That information cannot be extracted in the effective-sphere approximation, however, because fits to Eq. (2) impose azimuthal symmetry. Simulations such as the example in Fig. 3(a) confirm that effective-sphere characterization results are independent of $\phi$, which helps to explain why measurements of $\theta$ appear to be reliable.

\section{CONCLUSIONS}

We have shown that in-line holographic microscopy images of symmetric colloidal dimers can be interpreted with a generative model for ideal spherical scatterers to obtain estimates for the dimers' effective sizes, $d_{p}^{*}$, effec- 
tive refractive indexes, $n_{p}^{*}$, and axial positions relative to the focal plane, $z_{p}$. The effective-sphere diameter and refractive index are parameterized by a dimer's inclination, $\theta$, relative to the imaging plane in a way that can be computed using cluster T-matrix theory. The computed parameterization, in turn, can be used to measure the orientation of dimers in experimental data.

Effective-sphere analysis offers substantial benefits for analyzing colloidal dispersions including the availability of commercial instrumentation that can rapidly perform particle-resolved holographic characterization measurements on large statistical samples. When this technique is applied to nominally monodisperse colloidal dispersions, effective-sphere analysis usefully distinguishes dimers and other clusters from spheres by predicting the range of effective-sphere properties expected for dimers. Differentiating monomers and dimers in this way can be used to monitor the rate of aggregation in colloidal dispersions, with immediate application to label-free medical diagnostic testing based on detection of colloidal agglutination.

Within the distribution of identified dimers, effectivesphere analysis yields remarkably accurate results for the dimers' three-dimensional trajectories and also, apparently, for their three-dimensional orientations. These observations suggest that holographic particle characterization will be useful for studying the transport properties of aspherical colloids, including both rods and clusters, without requiring detailed fits to more descriptive theories of light scattering. This approach may prove fruitful for addressing outstanding questions about aspherical particles' tumbling in shear flows. It also should be useful for microrheology with anisotropic probe particles and for studying the interactions between aspherical objects at the micrometer scale. The ability to track three-dimensional trajectories and orientations in nearreal-time also should be useful for studies of active col- loidal particles [45] and bacteria [36], many of which are aspherical.

Planned extensions to this work include studies of more general types of colloidal clusters including asymmetric colloidal dimers, colloidal trimers and higher-order clusters. Effective-sphere analysis also should be useful for initializing fits to more complete theories for scattering by clusters [19, 20]. All such present and prospective applications build on the foundation of commercial and open-source instrumentation and software for holographic particle characterization to provide a new window onto the properties and dynamics of aspherical colloidal particles and their dispersions.

The data and open-source software used for this study are available online at https://github.com/laltman2/ Dimer_HVM.

\section{CONFLICTS OF INTEREST}

F.C.C. is a co-founder and Chief Technology Officer of Spheryx, Inc., the company that manufactures the xSight instrument for holographic particle characterization used in this study. D.G.G. is a co-founder of Spheryx, Inc.

\section{ACKNOWLEDGMENTS}

This work was supported principally by the RAPID program of the National Science Foundation through Award No. DMR-2027013. Additional funding was provided by the MRSEC program of the National Science Foundation through Award Number DMR-1420073. The Spheryx xSight used for this study was purchased by the NYU MRSEC as shared instrumentation.

We are grateful to Andrew Hollingsworth for providing the custom-synthesized colloidal silica spheres that were used in this study. We also are grateful to Alexander Y. Grosberg and Aleksander Donev for helpful conversations regarding Jeffery orbits.
[1] S.-H. Lee, Y. Roichman, G.-R. Yi, S.-H. Kim, S.-M. Yang, A. van Blaaderen, P. van Oostrum, and D. G. Grier, Characterizing and tracking single colloidal particles with video holographic microscopy, Opt. Express 15, 18275 (2007).

[2] K. Xiao and D. G. Grier, Multidimensional optical fractionation of colloidal particles with holographic verification, Phys. Rev. Lett. 104, 028302 (2010).

[3] C. Wang, F. C. Cheong, D. B. Ruffner, X. Zhong, M. D. Ward, and D. G. Grier, Holographic characterization of colloidal fractal aggregates, Soft Matter 12, 8774 (2016).

[4] C. Middleton, M. D. Hannel, A. D. Hollingsworth, D. J. Pine, and D. G. Grier, Optimizing the synthesis of monodisperse colloidal spheres using holographic particle characterization, Langmuir 35, 6602 (2019).

[5] Y. Zagzag, M. F. Soddu, A. D. Hollingsworth, and D. G. Grier, Holographic molecular binding assays, Sci. Rep. 10, 1 (2020).
[6] C. Wang, X. Zhong, D. B. Ruffner, A. Stutt, L. A. Philips, M. D. Ward, and D. G. Grier, Holographic characterization of protein aggregates, J. Pharm. Sci. 105, 1074 (2016).

[7] K. Snyder, R. Quddus, A. D. Hollingsworth, K. Kirshenbaum, and D. G. Grier, Holographic immunoassays: direct detection of antibodies binding to colloidal spheres, Soft Matter 16, 10180 (2020).

[8] J. Sheng, E. Malkiel, and J. Katz, Digital holographic microscope for measuring three-dimensional particle distributions and motions, Appl. Opt. 45, 3893 (2006).

[9] C. F. Bohren and D. R. Huffman, Absorption and Scattering of Light by Small Particles (Wiley, 1983).

[10] M. I. Mishchenko, L. D. Travis, and A. A. Lacis, Scattering, Absorption, and Emission of Light by Small Particles (Cambridge University Press, 2002).

[11] G. Gouesbet and G. Gréhan, Generalized Lorenz-Mie Theories (Springer, 2011). 
[12] M. Hannel, C. Middleton, and D. G. Grier, Holographic characterization of imperfect colloidal spheres, Appl. Phys. Lett. 107, 141905 (2015).

[13] F. C. Cheong, K. Xiao, D. J. Pine, and D. G. Grier, Holographic characterization of individual colloidal spheres' porosities, Soft Matter 7, 6816 (2011).

[14] M. A. Odete, F. C. Cheong, A. Winters, J. J. Elliott, L. A. Philips, and D. G. Grier, The role of the medium in the effective-sphere interpretation of holographic particle characterization data, Soft Matter 16, 891 (2020).

[15] L. E. Altman and D. G. Grier, Interpreting holographic molecular binding assays with effective medium theory, Biomed. Opt. Express 11, 5225 (2020).

[16] F. C. Cheong, P. Kasimbeg, D. B. Ruffner, E. H. Hlaing, J. M. Blusewicz, L. A. Philips, and D. G. Grier, Holographic characterization of colloidal particles in turbid media, Appl. Phys. Lett. 111, 153702 (2017).

[17] J. Fung and S. Hoang, Computational assessment of an effective-sphere model for characterizing colloidal fractal aggregates with holographic microscopy, J. Quant. Spectrosc. Radiat. Transf. 236, 106591 (2019).

[18] V. A. Markel, Introduction to the Maxwell Garnett approximation: tutorial, JOSA A 33, 1244 (2016).

[19] J. Fung, R. W. Perry, T. G. Dimiduk, and V. N. Manoharan, Imaging multiple colloidal particles by fitting electromagnetic scattering solutions to digital holograms, J. Quant. Spectrosc. Radiat. Transf. 113, 2482 (2012).

[20] J. Fung and V. N. Manoharan, Holographic measurements of anisotropic three-dimensional diffusion of colloidal clusters, Phys. Rev. E 88, 020302 (2013).

[21] W. J. Wiscombe, Improved Mie scattering algorithms, Appl. Opt. 19, 1505 (1980).

[22] W. Yang, Improved recursive algorithm for light scattering by a multilayered sphere, Appl. Opt. 42, 1710 (2003).

[23] O. Peña and U. Pal, Scattering of electromagnetic radiation by a multilayered sphere, Comp. Phys. Commun. 180, 2348 (2009).

[24] B. J. Krishnatreya and D. G. Grier, Fast feature identification for holographic tracking: The orientation alignment transform, Opt. Express 22, 12773 (2014).

[25] L. E. Altman and D. G. Grier, CATCH: Characterizing and tracking colloids holographically using deep neural networks, J. Phys. Chem. B 124, 1602 (2020).

[26] S.-H. Lee and D. G. Grier, Holographic microscopy of holographically trapped three-dimensional structures, Opt. Express 15, 1505 (2007).

[27] H. Shpaisman, B. Jyoti Krishnatreya, and D. G. Grier, Holographic microrefractometer, Appl. Phys. Lett. 101, 091102 (2012).

[28] F. C. Cheong, B. Sun, R. Dreyfus, J. Amato-Grill, K. Xiao, L. Dixon, and D. G. Grier, Flow visualization and flow cytometry with holographic video microscopy, Opt. Express 17, 13071 (2009).

[29] A. Yevick, M. Hannel, and D. G. Grier, Machine-learning approach to holographic particle characterization, Opt. Express 22, 26884 (2014).

[30] S. Barkley, T. Dimiduk, J. Fung, D. Kaz, V. N. Manoharan, R. McGorty, R. Perry, and A. Wang, Holographic microscopy with python and HoloPy, Comput. Sci. Eng.
1 (2019).

[31] D. Alves, R. Curvello, E. Henderson, V. Kesarwani, J. A. Walker, S. C. Leguizamon, H. McLiesh, V. S. Raghuwanshi, H. Samadian, E. M. Wood, Z. K. McQuilten, M. Graham, M. Wieringa, T. M. Korman, T. F. Scott, M. M. Banaszak Holl, G. Garnier, and S. R. Corrie, Rapid gel card agglutination assays for serological analysis following sars-cov-2 infection in humans, ACS Sensors 5, 2596 (2020).

[32] D. W. Mackowski and M. I. Mishchenko, Calculation of the $\mathrm{t}$ matrix and the scattering matrix for ensembles of spheres, J. Opt. Soc. Am. A 13, 2266 (1996).

[33] D. Mackowski and M. Mishchenko, A multiple sphere Tmatrix Fortran code for use on parallel computer clusters, J. Quant. Spectrosc. Radiat. Transf. 112, 2182 (2011).

[34] B. J. Krishnatreya, A. Colen-Landy, P. Hasebe, B. A. Bell, J. R. Jones, A. Sunda-Meya, and D. G. Grier, Measuring Boltzmann's constant through holographic video microscopy of a single sphere, Am. J. Phys. 82, 23 (2014).

[35] D. B. Ruffner, F. C. Cheong, J. M. Blusewicz, and L. A. Philips, Lifting degeneracy in holographic characterization of colloidal particles using multi-color imaging, Opt. Express 26, 13239 (2018).

[36] F. C. Cheong, C. C. Wong, Y. Gao, M. H. Nai, Y. Cui, S. Park, L. J. Kenney, and C. T. Lim, Rapid, highthroughput tracking of bacterial motility in $3 \mathrm{~d}$ via phasecontrast holographic video microscopy, Biophys. J. 108, 1248 (2015).

[37] F. Pedregosa, G. Varoquaux, A. Gramfort, V. Michel, B. Thirion, O. Grisel, M. Blondel, P. Prettenhofer, R. Weiss, V. Dubourg, J. Vanderplas, A. Passos, D. Cournapeau, M. Brucher, M. Perrot, and E. Duchesnay, Scikit-learn: Machine learning in Python, J. Mach. Learn. Res. 12, 2825 (2011).

[38] A. Zöttl, K. E. Klop, A. K. Balin, Y. Gao, J. M. Yeomans, and D. G. Aarts, Dynamics of individual brownian rods in a microchannel flow, Soft Matter 15, 5810 (2019).

[39] M. Frank, D. Anderson, E. R. Weeks, and J. F. Morris, Particle migration in pressure-driven flow of a brownian suspension, J. Fluid Mech. 493, 363 (2003).

[40] G. B. Jeffery, The motion of ellipsoidal particles immersed in a viscous fluid, Proc. Roy. Soc. London A 102, 161 (1922).

[41] J. Einarsson, F. Candelier, F. Lundell, J. R. Angilella, and B. Mehlig, Rotation of a spheroid in a simple shear at small reynolds number, Physics of Fluids 27, 063301 (2015)

[42] S. Kim and S. J. Karrila, Microhydrodynamics: Principles and Selected Applications (Courier Corporation, 2013).

[43] M. Hoffmann, C. S. Wagner, L. Harnau, and A. Wittemann, 3D Brownian diffusion of submicron-sized particle clusters, ACS Nano 3, 3326 (2009).

[44] W. Srinin, Non-local and Non-linear Rheology of Polymer Rings, Ph.D. thesis, New York University (2019).

[45] F. Ma, X. Yang, H. Zhao, and N. Wu, Inducing propulsion of colloidal dimers by breaking the symmetry in electrohydrodynamic flow, Phys. Rev. Lett. 115, 208302 (2015). 